\title{
INVERSION DE LAPLACE EFFECTIVE
}

\section{By ANDRÉ STEF AND GÉRALd TENENBAum \\ Université Henri Poincaré-Nancy 1}

Let $F, G$ be arbitrary distribution functions on the real line and let $\widehat{F}, \widehat{G}$ denote their respective bilateral Laplace transforms. Let $\kappa>0$ and let $h: \mathbb{R}^{+} \rightarrow \mathbb{R}^{+}$be continuous, non-decreasing, and such that $h(u) \geq A u^{4}$ for some $A>0$ and all $u \geq 0$. Under the assumptions that

$$
\sup _{0 \leq u \leq \kappa}|\widehat{F}(u)-\widehat{G}(u)| \leq \varepsilon, \quad \widehat{F}(u)+\widehat{G}(u) \leq h(u) \quad(-L \leq u \leq L),
$$

we establish the bound

$$
\sup _{u \in \mathbb{R}}|\widehat{F}(u)-\widehat{G}(u)| \leq C Q_{G}(l)
$$

where $C$ is a constant depending at most on $\kappa$ and $A, Q_{G}$ is the concentration function of $G$, and $l:=(\log L) / L+(\log W) / W$, with $W$ any solution to $h(W)=1 / \varepsilon$. Improving and generalizing an estimate of Alladi, this result provides a Laplace transform analogue to the Berry-Esseen inequality, related to Fourier transforms. The dependence in $\varepsilon$ is optimal up to the logarithmic factor $\log W$. A number-theoretic application, developed in detail elsewhere, is described. It concerns so-called lexicographic integers, whose characterizing property is that their divisors are ranked according to size and valuation of the largest prime factor. The above inequality furnishes, among other informations, an effective Erdös-Kac theorem for lexicographical integers.

1. Introduction. Soient $F$ et $G$ deux fonctions de répartition sur $\mathbb{R}$. Notons

$$
f(\tau):=\int_{\mathbb{R}} \mathrm{e}^{i \tau z} \mathrm{~d} F(z) \quad(\tau \in \mathbb{R})
$$

la fonction caractéristique, ou transformée de Fourier-Stieltjes, de $F$, et $g(\tau)$ celle de $G$. L'inégalité classique de Berry-Esseen [3], [9], est l'outil de base de l'inversion de Fourier effective. Elle permet de majorer la quantité

$$
\|F-G\|_{\infty}:=\sup _{z \in \mathbb{R}}|F(z)-G(z)|
$$

en termes d'une estimation de $|f-g|$ en moyenne et d'une information quantitative sur la régularité de $G$. L'énoncé usuel se place dans l'hypothèse d'absolue continuité de l'une des deux fonctions de répartition, par exemple $G$ (cf., par exemple, Feller [10], chapitre XVI.3, lemma 2), mais la même technique fournit un résultat en toute généralité, exprimé à l'aide de la fonction de concentration

$$
Q_{G}(l):=\sup _{z}\{G(z+l)-G(z)\} \quad(l>0) .
$$

Received May 1999.

AMS 2000 subject classifications. Primary 60E10; secondary 11N25, 40E05, 44A10.

Key words and phrases. Laplace transform, effective inversion, Berry-Esseen inequality, concentration functions, one-sided $L^{1}$-approximation, lexicographical integers. 
Elliott ([5], lemma 1.47) montre ainsi que la majoration ${ }^{1}$

$$
\|F-G\|_{\infty} \ll Q_{G}\left(\frac{1}{T}\right)+\int_{-T}^{T}\left|\frac{f(\tau)-g(\tau)}{\tau}\right| \mathrm{d} \tau
$$

est valable, uniformément en $F$ et $G$, pour toute valeur du nombre réel positif $T .^{2}$

Il est naturel de se demander s'il existe une formule d'inversion quantitative analogue à (1.2) pour la transformation de Laplace. L'intérêt analytique d'une telle estimation est patent: le fait que la transformée d'une mesure positive soit réelle positive pour des valeurs réelles de la variable ouvre la possibilité d'employer des techniques d'approximation spécifiques impraticables dans le cadre de la transformation de Fourier.

Cette étude a été entreprise par Alladi dans [1], [2]. Comme la transformée de Laplace d'une fonction de répartition

$$
\widehat{F}(s):=\int_{\mathbb{R}} \mathrm{e}^{-s z} \mathrm{~d} F(z)
$$

est analytique dans son domaine de convergence, l'approximation sur un ensemble de valeurs de $s$ ayant un point d'accumulation constitue une notion pertinente de distance. Avec des applications arithmétiques en vue, Alladi [2] a choisi des hypothèses de majoration de $|\widehat{F}(u)-\widehat{G}(u)|$ pour $u$ variant dans un petit intervalle réel dont l'origine est une extrémité. Il montre en particulier dans [2] le résultat suivant, où l'on note

$$
\Phi(z):=\frac{1}{\sqrt{2 \pi}} \int_{-\infty}^{z} \mathrm{e}^{-u^{2} / 2} \mathrm{~d} u
$$

la fonction de répartition de la loi de Gauss.

ThÉORÈme A (Alladi). Soit $F$ une fonction de répartition. Soient $\varepsilon, \kappa, L$ des nombres réels satisfaisant à $0<\varepsilon<1 / 3,0<\kappa \leq L$, et tels que:

(i) $\left|\widehat{F}(u)-\mathrm{e}^{u^{2} / 2}\right| \leq \varepsilon \quad(0 \leq u \leq \kappa)$,

(ii) $\widehat{F}(u) \ll \mathrm{e}^{u^{2} / 2} \quad(-L \leq u \leq L)$.

On a alors

$$
\|F-\Phi\|_{\infty} \ll_{\kappa} \frac{1}{\sqrt{L}}+\left(\frac{\log |\log \varepsilon|}{|\log \varepsilon|}\right)^{1 / 8}
$$

\footnotetext{
${ }^{1}$ Ici et dans la suite nous employons la notation de Vinogradov $f \ll g$ pour signifier l'existence d'une constante $C$ positive, qui peut être absolue ou dépendre de certains paramètres, telle que $|f| \leq C|g|$ dans le domaine de définition commun aux fonctions $f$ et $g$.

${ }^{2}$ Cette inégalité ne fournit une borne finie que si $|f-g|$ est intégrable pour la mesure $\mathrm{d} \tau / \tau$. Une condition suffisante pour cela est que $f$ et $g$ appartiennent à l'espace $E$ des fonctions $\varphi$ telles que $|1-\varphi(\tau)| /|\tau|$ soit intégrable à l'origine. Il n'est pas difficile de montrer qu'une fonction caractéristique $\varphi$ est dans $E$ si et seulement si sa fonction originale $H$ vérifie
}

$$
\int_{\mathbb{R}} \log ^{+}|z| \mathrm{d} H(z)<\infty .
$$


Nous nous proposons dans cet article d'établir un résultat qui généralise et précise celui d'Alladi. L'approximation de $F$ n'est plus nécessairement gaussienne et peut être une fonction de répartition quelconque, $G$. De plus, afin d'assouplir le cadre d'application, nous introduisons une fonction auxiliaire $h$, continue croissante sur $\mathbb{R}^{+}$, qui joue le rôle de la fonction majorante dans la condition (ii) du Théorème A. En pratique, on pourra fréquemment choisir $h=h_{G}$ avec $h_{G}(u):=\widehat{G}(u)+\widehat{G}(-u)$. Nous imposons la condition

$$
h(u) \gg u^{4} \quad(u \geq 0)
$$

qui est une conséquence immédiate des hypothèses dès que l'un au moins des supports de $F$ ou $G$ n'est pas réduit à l'origine.

THÉORÈme. Soient $F, G$ deux fonctions de répartition, et $h: \mathbb{R}^{+} \rightarrow \mathbb{R}^{+}$une fonction continue croissante satisfaisant à la condition (1.3). Soient $\varepsilon, \kappa, L$ des nombres réels satisfaisant à $0<\varepsilon<1 /\{3+h(2)\}, 0<\kappa \leq L$, et tels que:

(i) $|\widehat{F}(u)-\widehat{G}(u)| \leq \varepsilon \quad(0 \leq u \leq \kappa)$,

(ii) $\widehat{F}(u)+\widehat{G}(u) \ll h(|u|)<\infty \quad(-L \leq u \leq L)$.

Alors on a

$$
\|F-G\|_{\infty} \ll Q_{G}\left(\frac{\log L}{L}+\frac{\log W}{W}\right),
$$

où $W$ est une solution quelconque de l'équation $h(W)=1 / \varepsilon$. La constante implicite dans (1.4) dépend au plus de $\kappa$ et des constantes implicites de (1.3) et (ii).

Nous mentionnons pour mémoire la reformulation de notre théorème dans le cadre de la transformation de Mellin. On obtient, pour toutes fonctions de répartition, $F, G$ sur $\mathbb{R}^{+}$et sous les hypothèses

$$
\left\{\begin{array}{l}
\left|\int_{0}^{\infty} t^{-s}\{\mathrm{~d} F(t)-\mathrm{d} G(t)\}\right| \leq \varepsilon \quad(0 \leq s \leq \kappa), \\
\int_{0}^{\infty} t^{-s}\{\mathrm{~d} F(t)+\mathrm{d} G(t)\} \ll h(|s|) \quad(-L \leq s \leq L),
\end{array}\right.
$$

l'estimation

$$
\|F-G\|_{\infty} \ll M_{G}\left(\frac{\log L}{L}+\frac{\log W}{W}\right),
$$

où l'on a posé $M_{G}(l):=\sup _{t>0}\{G(t+l t)-G(t)\}$.

Le résultat suivant est une application de (1.4) dans le cas particulier où $G$ est absolument continue et à densité bornée.

COROLlaire. On conserve les hypothèses du Théorème et l'on suppose en outre que:

(iii) G est absolument continue avec $G^{\prime} \in L^{\infty}(\mathbb{R})$;

(iv) $\exists \vartheta \geq 1: \log h(u) \ll 1+u^{\vartheta}(0 \leq u \leq L)$. 
Alors

$$
\|F-G\|_{\infty} \ll\left(\frac{\log L}{L}+\frac{\log |\log \varepsilon|}{|\log \varepsilon|^{1 / \vartheta}}\right)\left\|G^{\prime}\right\|_{\infty}
$$

où la constante implicite dépend au plus de $\kappa$ et des constantes implicites de (ii) et (iv).

L'exposant de $|\log \varepsilon|$ apparaissant dans (1.6) est optimal, au moins lorsque $\vartheta=2 k /(2 k-1)\left(k \in \mathbb{N}^{*}\right)$. Nous construisons au paragraphe 3 une suite de contre-exemples explicites.

Nous terminons cette introduction par une remarque relative à certaines extensions connues de l'inégalité de Berry-Esseen-voir, par exemple, Petrov [17], lemme VI.2.8, théorème VI.3.2. Ces théorèmes fournissent, pour chaque nombre réel $z$, une majoration de $|F(z)-G(z)|$ qui tend vers 0 lorsque $|z| \rightarrow \infty$ et qui, sous des hypothèses convenables de régularité de $G$, demeure cependant uniformément petite dès que $|g-f|$ est petite pour une norme en moyenne adéquate. Bien que la méthode du présent travail soit également susceptible de fournir des analogues de ces résultats locaux dans le cadre de la transformation de Laplace, nous n'avons pas cherché ici à établir de telles estimations.

2. Applications. Nous donnons ici une brève description des possibilités d'application de notre résultat dans un contexte de théorie analytique des nombres.

Soit $\omega(n)$ le nombre des facteurs premiers d'un entier $n$, comptés sans multiplicité. Le célèbre théorème d'Erdős et Kac [7], [8], énonce que la suite de fonctions de répartition ${ }^{3}$

$$
F_{N}(z):=\frac{1}{N}\left|\left\{n: \omega(n) \leq \log _{2} N+z \sqrt{\log _{2} N}\right\}\right|
$$

converge faiblement vers la loi de Gauss. L'estimation optimale du terme d'erreur a été conjecturée par LeVeque en 1949, et établie par Rényi et Turán en $1959 .{ }^{4}$ Leur démonstration de la formule uniforme en $z \in \mathbb{R}, N \geq 3$,

$$
F_{N}(z)=\Phi(z)+O\left(\frac{1}{\sqrt{\log _{2} N}}\right),
$$

est aujourd'hui classique, ${ }^{5}$ et repose sur l'inégalité de Berry-Esseen, via une évaluation adéquate de la transformée de Fourier

$$
\varphi_{N}(\vartheta):=\frac{1}{N} \sum_{n \leq N} \exp \left\{i \vartheta \frac{\omega(n)-\log _{2} N}{\sqrt{\log _{2} N}}\right\} \text {. }
$$

\footnotetext{
${ }^{3}$ Ici et dans la suite $\log _{k}$ désigne la $k$-ième itérée de la fonction logarithme.

${ }^{4}$ L'optimalité peut être établie de diverses manières. Pour une évaluation asymptotique du terme résiduel, voir Delange [4].

${ }^{5}$ Voir par exemple [21], § III.4.4.
} 
Cependant, Rényi et Turán mentionnent dans leur article que l'idée de cette preuve remonte à la thèse de Turán [22], publiée en hongrois en 1934. Elliott reproduit dans [5], pages 18-20, une fascinante lettre de Turán datant de 1976 dans laquelle il explique qu'il s'est approché, dans sa thèse, d'une preuve que les $F_{N}$ ont une limite gaussienne en montrant que l'on a uniformément pour $-\frac{1}{2} \leq r \leq \frac{1}{2}$

$$
\sum_{n \leq N} 2^{r \omega(n)}=\{1+o(1)\} D(r) N(\log N)^{2^{r}-1},
$$

avec une constante convenable $D(r)$. Il décrit, dans un premier temps, comment il avait initialement appliqué (2.1) à une nouvelle preuve du théorème de Hardy et Ramanujan. ${ }^{6}$ Ensuite, il évoque ses conversations avec Rényi de 1956-57, où il exprimait le sentiment que (2.1) devait contenir une plus grande quantité d'information. Rényi observa rapidement qu'une extension (d'ailleurs triviale) de (2.1) aux valeurs complexes de $r$ - en fait $r=i \vartheta / \sqrt{\log _{2} N}$-suffisait, grâce à la théorie des fonctions caractéristiques, à impliquer le théorème d'Erdős et Kac.

Notre théorème montre que Turán était encore plus près qu'il ne l'imaginait d'une preuve du mythique théorème d'Erdős-Kac: quand on choisit

$$
r \log 2=-u / \sqrt{\log _{2} N}
$$

le membre de gauche de (2.1) n'est autre que

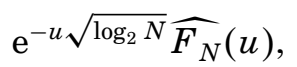

et une application immédiate de (1.4) permet d'inférer directement de (2.1) que

$$
F_{N}(z)=\Phi(z)+o(1) \quad(N \rightarrow \infty)
$$

uniformément en $z \in \mathbb{R}$. En réalité, moyennant les faits relativement aisés à établir que le terme d'erreur de (2.1) est $\ll 1 / \log N$ et que $D(r)=1+O(r)$ quand $r \rightarrow 0$, un calcul élémentaire fournit

$$
\widehat{F_{N}}(u)=\mathrm{e}^{u^{2} / 2}\left\{1+O\left(\frac{u}{\sqrt{\log _{2} N}}+\frac{1}{\log N}\right)\right\} \quad\left(|u| \leq \sqrt{\log _{2} N}\right) .
$$

La relation (1.4) avec $L=\sqrt{\log _{2} N}, \varepsilon=1 / L$ et $\kappa=1$ implique donc que le terme d'erreur de (2.2) est

$$
\ll \log _{4} N / \sqrt{\log _{3} N} .
$$

Cette estimation représente, au facteur $\log _{4} N$ près, le meilleur résultat quantitatif déductible d'une formule réelle de type (2.1) pour un théorème d'ErdősKac effectif.

Dans la mesure où la formule (2.1) n'est pas plus difficile à obtenir pour des valeurs complexes que pour des valeurs réelles de la variable, l'intérêt

${ }^{6}$ C'est-à-dire l'assertion que $\omega(n) \sim \log _{2} N$ pour $N+o(N)$ entiers $n \leq N$. 
d'appliquer une technique d'inversion de Laplace effective dans ce problème est essentiellement historique et méthodologique. Cependant, la situation possède également une intrinsèque valeur d'exemplarité: dans certaines questions conduisant à des évaluations asymptotiques similaires, l'emploi des variables complexes est délicat, voire impossible, alors même qu'on dispose de méthodes efficaces lorsque les variables sont réelles. Nous nous proposons de décrire plus précisément une situation de ce type que nous avons rencontrée récemment - et qui a d'ailleurs constitué la motivation initiale de la présente étude.

Posons $d(n ; w):=\max \left\{d: d \mid n, P^{+}(d)<w\right\}$ où $P^{+}(d)$ désigne le plus grand facteur premier de $d$ avec la convention $P^{+}(1)=\infty$. La croissance normale des facteurs premiers d'un entier étant doublement exponentielle (cf. [6], [12], chapitre 1$)$, on peut s'attendre à ce que $\log d(n ; w)$ soit usuellement comparable à $\log w$ et de nombreux travaux de théorie probabiliste des nombres utilisent cette propriété. Nous avons abordé dans [20] l'étude de la répartition de la fonction arithmétique ${ }^{7}$

$$
W(n):=\min _{p \mid n} p / d(n ; p),
$$

avec la convention $W(1)=\infty$.

Notant $\mathscr{L}(y)$ l'ensemble des entiers $n \geq 1$ tels que $W(n)>y$, nous avons désigné dans [20] l'ensemble $\mathscr{L}(1)$ comme l'ensemble des entiers lexicographiques. Cette dénomination est motivée par le fait qu'une condition nécessaire et suffisante pour qu'un entier

$$
n=\prod_{1 \leq j \leq k} p_{j}^{\nu_{j}}
$$

avec $p_{1}<\cdots<p_{k}$, soit dans $\mathscr{L}(1)$ est que ses diviseurs soient rangés dans l'ordre lexicographique, obtenu en associant à chaque diviseur

$$
d=\prod_{1 \leq j \leq k} p_{j}^{\alpha_{j}} \quad\left(0 \leq \alpha_{j} \leq \nu_{j}\right)
$$

le mot $\alpha_{k} \cdots \alpha_{1}$ de longueur $k=\omega(n)$.

Une seconde propriété attrayante de l'ensemble $\mathscr{L}(1)$, également établie dans [20], est que la relation

$$
W(n)=\min _{1 \leq j<\tau(n)} d_{j+1}(n) / d_{j}(n),
$$

où $\left\{d_{j}(n)\right\}_{j=1}^{\tau(n)}$ désigne la suite croissante des diviseurs de $n$, a lieu si, et seulement si, $n \in \mathscr{L}(1)$.

Soit $\mathscr{E}$ l'ensemble des entiers $n$ tels que

$$
\min _{1 \leq j<\tau(n)} d_{j+1}(n) / d_{j}(n) \geq 2 .
$$

\footnotetext{
${ }^{7}$ Ici et dans la suite, nous réservons la lettre $p$ pour désigner un nombre premier.
} 
On a donc $\mathscr{L}(2) \subset \mathscr{E}$. Une ancienne conjecture d'Erdős, établie par Maier et Tenenbaum en 1984 [16], affirme que $\mathscr{E}$ est de densité nulle, autrement dit

$$
E(x):=|\mathscr{E} \cap[1, x]|=o(x) \quad(x \rightarrow \infty) .
$$

Le meilleur encadrement actuellement connu pour $E(x)$ est dû à Stef [19], qui établit que l'on a pour une constante positive convenable $c$

$$
x(\log x)^{-\beta+o(1)} \ll E(x) \ll x \mathrm{e}^{-c \sqrt{\log _{2} x}},
$$

avec $\beta=1-\left(1+\log _{2} 3\right) / \log 3 \approx 0,00415$.

Ces estimations reflètent l'état actuel de nos connaissances sur la répartition des entiers selon les diverses structures multiplicatives possibles. Nous renvoyons le lecteur à [20] pour de plus amples commentaires à ce sujet, et nous nous contentons de signaler que la minoration de (2.6) correspond essentiellement à un ensemble d'entiers $n$ tels que $\omega(n) \leq\left(\log _{2} n\right) / \log 3$. La notion d'entier lexicographique permet d'exhiber une autre structure multiplicative représentative d'une partie significative de $\mathscr{E}$, et, en particulier, de fournir une première minoration non triviale pour le nombre des entiers $n$ de $\mathscr{E}$ n'excédant pas $x$ et possédant le nombre "normal" de facteurs premiers, soit $\omega(n) \sim \log _{2} x$.

Posons $\mathscr{L}(x, y):=\mathscr{L}(y) \cap[1, x]$. Les motivations exposées plus haut nous conduisent à étudier le comportement asymptotique de la quantité

$$
L(x, y ; t):=\sum_{n \in \mathscr{L}(x, y)} t^{\omega(n)} .
$$

Pour $t>0$, soit $\delta=\delta(t)$ l'unique solution dans ] $-\infty, 1[$ de l'équation

$$
t \int_{0}^{1 / 2} \frac{\mathrm{d} v}{v^{\delta}(1-v)}=1
$$

Posons encore $\varrho(t):=1-\delta(t)$ et $\eta(t):=\min \left(\varrho(t), 1 / \varrho(t)^{2}\right)$. Nous avons montré dans [20] que, pour chaque $y \geq 1$, il existe une fonction

$$
K_{y} \in \mathscr{C}^{1}\left(\mathbb{R}^{+*}, \mathbb{R}^{+*}\right)
$$

telle que l'on ait

$$
L(x, y ; t)=\left\{K_{y}(t)+O_{T}\left(\frac{\left(\log _{2} x\right)^{2}}{(\log x)^{\eta(t)}}\right)\right\} \frac{x}{(\log x)^{\delta(t)}} \quad(x \geq 3)
$$

pour tout $T>1$ et uniformément dans le domaine $1 / T \leq t \leq T, 1 \leq y \leq T$.

Lorsque $t=1$, on obtient une formule asymptotique pour la fonction de comptage des entiers lexicographiques. On note que $\delta(1) \approx 0,2228$.

Posons $a(t):=-t \delta^{\prime}(t), b(t):=-t \delta^{\prime}(t)-t^{2} \delta^{\prime \prime}(t)>0, M=M(x ; t):=$ $a(t) \log _{2} x, V=V(x ; t):=b(t) \log _{2} x$, et

$$
F_{x, t}(z):=\frac{1}{L(x, y ; t)} \sum_{\substack{n \in \mathscr{\ell}(x, y) \\ \omega(n) \leq M+z \sqrt{V}}} t^{\omega(n)} .
$$


Un calcul de routine permet de déduire de (2.8), que, pour chaque $T>1$ fixé, on a uniformément pour $|u| \leq\left(\log _{2} x\right)^{\eta(t) / 8}, 1 / T \leq t \leq T, 1 \leq y \leq T$,

$$
\widehat{F_{x, t}}(u)=\left\{1+O\left(\frac{1}{\left(\log _{2} x\right)^{1 / 8}}\right)\right\} \mathrm{e}^{u^{2} / 2} .
$$

La formule (1.4) implique donc immédiatement

$$
F_{x, t}(z)=\Phi(z)+O\left(\frac{\log _{4} x}{\sqrt{\log _{3} x}}\right)
$$

uniformément pour $1 / T \leq t \leq T, 1 \leq y \leq T, z \in \mathbb{R}$.

On déduit de cette formule de nombreux renseignements, détaillés dans [20], sur la structure des nombres lexicographiques. Contentons-nous ici d'en indiquer deux. Tout d'abord, nous observons que la formule (2.10) avec $t=1$ constitue un théorème d'Erdős-Kac pour les entiers lexicographiques. L'ordre normal du nombre des facteurs premiers d'un entier lexicographique $n$ est $a \log _{2} n$ avec $a=-\delta^{\prime}(1) \approx 0,5624$ et la répartition statistique de $\omega(n)$ autour de cette valeur est asymptotiquement gaussienne, d'écart-type $\sqrt{b(1) \log _{2} n}$. Ensuite, si $\mu=\delta(s)+\log s \approx 0,687$ où $s$ est l'unique solution de $\delta^{\prime}(s)=-1$, on peut déduire de (2.10) que l'on a, pour chaque $z>0$,

$$
\sum_{\substack{n \in \mathscr{L}(x, y) \\\left|\omega(n)-\log _{2} x\right| \leq z \sqrt{\log _{2} x}}} 1=\frac{x}{(\log x)^{\mu}} \exp \left\{O\left(\sqrt{\log _{2} x}\right)\right\}
$$

uniformément pour $1 \leq y \leq T, x \geq 16$. Cela fournit une minoration effective de la taille du sous-ensemble de $\mathscr{E}$ constitué des entiers qui satisfont au théorème de Hardy-Ramanujan.

Du point de vue théorique, la situation est ici radicalement différente de celle du théorème d'Erdős-Kac précédemment décrite. En effet, l'évaluation (2.8) de la transformée de Laplace n'est plus obtenue par intégration complexe, susceptible de traiter indifféremment le cas d'une variable $t$ réelle ou complexe, mais via des équations fonctionnelles dont la résolution asymptotique impose, en l'état actuel des connaissances, un traitement par affinage inductif d'inégalités requérant la condition $t>0$. Ainsi, la formule effective (2.10) constitue essentiellement, en dépit de la relative faiblesse du terme résiduel, la limite naturelle ${ }^{8}$ des méthodes actuellement disponibles pour évaluer la vitesse de convergence vers la loi de Gauss des fonctions de répartition $F_{x, t}$.

3. Dépendance en $\varepsilon$. Considérons d'abord le cas $k=1$. Posons

$G(z):=\frac{1}{\sqrt{2 \pi}} \int_{-\infty}^{z} \mathrm{e}^{-t^{2} / 2} \mathrm{~d} t, \quad F_{n}(z):=\frac{1}{\sqrt{2 \pi}} \int_{-\infty}^{z}\{1+\sin (n t)\} \mathrm{e}^{-t^{2} / 2} \mathrm{~d} t \quad(n \in \mathbb{N})$.

On a bien $F_{n}(\infty)=1$ car, sur $\mathbb{R}$, l'intégrale relative au sinus est nulle par symétrie.

${ }^{8} \mathrm{Au}$ facteur $\log _{4} x$ près. 
On a classiquement

$$
\frac{1}{\sqrt{2 \pi}} \int_{-\infty}^{+\infty} \mathrm{e}^{-\left(z^{2} / 2\right)-u(z+i n)} \mathrm{d} z=\mathrm{e}^{\left(u^{2} / 2\right)+i u n-n^{2} / 2},
$$

donc

$$
\frac{1}{\sqrt{2 \pi}} \int_{-\infty}^{+\infty} \sin (n z) \mathrm{e}^{-z^{2} / 2} \mathrm{e}^{-u z} \mathrm{~d} z=-\sin (n u) \mathrm{e}^{u^{2} / 2} \mathrm{e}^{-n^{2} / 2} .
$$

Cela implique

$$
\widehat{F_{n}}(u)=\left\{1+O\left(\mathrm{e}^{-n^{2} / 2}\right)\right\} \widehat{G}(u) \quad(u \in \mathbb{R}) .
$$

On a par ailleurs

$$
\begin{aligned}
2\left\|F_{n}-G\right\|_{\infty} & \geq\left[F_{n}(z)-G(z)\right]_{\pi / 4 n}^{3 \pi / 4 n} \\
& =\frac{1}{\sqrt{2 \pi}} \int_{\pi / 4 n}^{3 \pi / 4 n} \sin (n u) \mathrm{e}^{-u^{2} / 2} \mathrm{~d} u \gg \frac{1}{n} .
\end{aligned}
$$

Choisissant $\varepsilon=\varepsilon_{n}:=\mathrm{e}^{-n^{2} / 2}$, on a donc simultanément, pour tout entier $n \geq 1$,

$$
\left|\widehat{F_{n}}(u)-\widehat{G}(u)\right| \ll \varepsilon_{n} \quad(0 \leq u \leq 1), \quad \text { et } \quad\left\|F_{n}-G\right\|_{\infty} \gg 1 / \sqrt{\left|\log \varepsilon_{n}\right|} .
$$

Cela établit bien l'optimalité de l'exposant $1 / \vartheta$ de (1.6) lorsque $\vartheta=2$.

Le cas général $\vartheta=2 k /(2 k-1)$ peut être traité de manière semblable, en considérant des lois dont les densités respectives sont proportionnelles à $\exp \left\{-t^{2 k} / 2 k\right\}$ et à $(1+\sin n t) \exp \left\{-t^{2 k} / 2 k\right\}$. Nous omettons les détails et nous nous contentons d'indiquer que l'analogue (restreint au domaine $|u| \leq 1$ ) de la formule (3.1) est fourni par le résultat auxiliaire suivant, relatif à la croissance de certaines transformées de Laplace.

Lemme 0. Soit $k \in \mathbb{N}^{*}$. Il existe une constante $\gamma_{k}>0$ telle que l'on ait

$$
\begin{aligned}
\Phi_{k}(u+i v) & :=\int_{\mathbb{R}} \mathrm{e}^{-\left(t^{2 k} / 2 k\right)+(i v+u) t} \mathrm{~d} t \\
& \ll \exp \left\{-\gamma_{k}|v|^{2 k /(2 k-1)}\right\} \quad(|u| \leq 1, v \in \mathbb{R}) .
\end{aligned}
$$

DÉmOnstration. On peut manifestement supposer $v>0$. Posons alors $v=s^{2 k-1}, u=s^{2 k-1} w_{s}$, et effectuons le changement de variable $t=s y$ dans l'intégrale de (3.2). Nous obtenons

$$
\Phi_{k}(u+i v)=s \int_{\mathbb{R}} \exp \left\{-s^{2 k}\left(g(y)-w_{s} y\right)\right\} \mathrm{d} y
$$

avec $g(y):=\left(y^{2 k} / 2 k\right)-i y$. Cette intégrale relève de la méthode du col. Nous considérons le terme $w_{s} y$ comme une petite perturbation et traitons $g(y)$ comme un terme principal. Les points critiques sont alors donnés par 
l'équation $y^{2 k-1}=i$. Les deux solutions les plus proches de l'axe réel sont $\mathrm{e}^{i \alpha_{k}}$ et $-\mathrm{e}^{-i \alpha_{k}}$, avec la notation

$$
\alpha_{k}:=\pi /(4 k-2) .
$$

Déplaçons la droite d'intégration jusqu'à $\Im m y=\sin \alpha_{k}$ : il est immédiat que cela n'altère pas la valeur de l'intégrale. Il suit

$$
\left|\Phi_{k}(u+i v)\right| \leq 2 s \int_{0}^{\infty} \exp \left\{-s^{2 k}\left(f(z)-z\left|w_{s}\right|\right)\right\} \mathrm{d} z
$$

avec $f(z):=\Re e g\left(z+i \sin \alpha_{k}\right)=\sin \alpha_{k}+\Re e\left(z+i \sin \alpha_{k}\right)^{2 k} / 2 k$. On a clairement $f(z) \sim z^{2 k} / 2 k$ lorsque $z \rightarrow \infty$, la majoration requise résulte donc de l'inégalité

$$
\inf _{z \geq 0} f(z)>0,
$$

que nous allons maintenant établir.

Lorsque $z \geq \cos \alpha_{k}$, on a $\arg \left(z+i \sin \alpha_{k}\right) \leq \alpha_{k} \leq \pi / 2 k$, donc

$$
\Re e\left(z+i \sin \alpha_{k}\right)^{2 k} \geq \cos \left(2 k \alpha_{k}\right)=-\sin \alpha_{k} .
$$

Cela implique $f(z) \geq(1-1 / 2 k) \sin \alpha_{k}$ pour ces valeurs de $z$.

Lorsque $0 \leq z \leq \cos \alpha_{k}$, on a

$$
\frac{1}{2 k}\left|\Re e\left(z+i \sin \alpha_{k}\right)^{2 k}\right| \leq \frac{1}{2 k},
$$

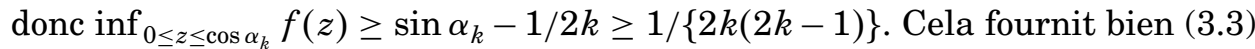
et achève la preuve du lemme.

4. Estimations auxiliaires. Nous étendons ici la définition (1.1) à toute fonction croissante $G$. Nous aurons à plusieurs reprises l'occasion d'utiliser l'estimation triviale

$$
Q_{G}(\alpha l) \leq\lceil\alpha\rceil Q_{G}(l) \quad(\alpha>0, l>0),
$$

où $\lceil\alpha\rceil$ désigne, pour $\alpha \in \mathbb{R}$, le plus petit entier au moins égal à $\alpha$.

La preuve de notre théorème repose essentiellement sur un lemme d'approximation unilatérale, en norme $L^{1}(\mathrm{~d} F)$, des fonctions à variation bornée sur $[-1,1]{ }^{9}$ Ce résultat étend aux mesures de probabilité $\mathrm{d} F(u)$ un résultat de Freud [11] relatif à la mesure de Lebesgue $\mathrm{d} u$. La preuve autonome que nous en donnons suit celle du théorème II.7.10 de [21], elle-même adaptée du lemme 2.1 de Korevaar [14].

Nous définissons la longueur d'un polynôme $P(x):=\sum_{m=0}^{n} a_{m} x^{m}$ par

$$
l(P):=\sum_{0 \leq m \leq n}\left|a_{m}\right|,
$$

et nous désignons par $Y(z):=\mathbf{1}_{[0, \infty[}(z)$ l'échelon unité de Heaviside.

\footnotetext{
${ }^{9}$ Ainsi qu'on le verra, seul un cas particulier de ce lemme nous sera nécessaire. Nous énonçons le cas général à fins de référence ultérieure.
} 
LEMME 1. Soit $f$ une fonction à variation bornée sur $[-1,1]$. Il existe une constante $A>0$, ne dépendant que de $f$, telle que, pour chaque entier $n \geq 1$, il existe des polynômes $R, S$, de degrés $\leq n$ vérifiant, pour toute fonction croissante $F:[-1,1] \rightarrow \mathbb{R}$, le système de relations:

$$
\left\{\begin{array}{l}
R(z) \leq f(z) \leq S(z) \quad(z \in \mathbb{R}), \\
l(R)+l(S) \leq A^{n}, \\
\int_{-1}^{1}\{S(z)-R(z)\} \mathrm{d} F(z) \ll Q_{F}(1 / n) .
\end{array}\right.
$$

DÉMONSTRATION. Nous pouvons nous restreindre au cas $f(z)=Y(z)$ : le cas général en résulte par application de la formule

$$
f(z)=f(-1)+\int_{-1}^{1} Y\left(\frac{1}{2} z-\frac{1}{2} \xi\right) \mathrm{d} f_{1}(\xi)-\int_{-1}^{1} Y\left(\frac{1}{2} z-\frac{1}{2} \xi\right) \mathrm{d} f_{2}(\xi),
$$

où $f_{1}, f_{2}$ sont des fonctions croissantes dont la différence est $f$. Nous omettons les détails.

Le cas $n=1$ est trivial puisque le choix $R=0, S=1$ vérifie alors le système (4.2). Lorsque $n \geq 2$, on peut supposer $n \equiv 2(\bmod 4)$. Nous posons alors $n=2 r-2$ avec $r=2 m$ et nous introduisons, ainsi qu'il est usuel dans ce type de problème, le polynôme de Tchébychev de degré $r$, soit $T_{r}(x)$, défini sur $[-1,1]$ par

$$
T_{r}(x):=\cos (r u) \quad(x=\cos u) .
$$

On a

$$
T_{r}(x)=2^{r-1} \prod_{\nu=1}^{r}\left(x-x_{\nu}\right)=2^{r-1} \prod_{\nu=1}^{m}\left(x^{2}-x_{\nu}^{2}\right),
$$

où l'on a posé $x_{\nu}:=\cos \left(\pi\left(\nu-\frac{1}{2}\right) / r\right)(1 \leq \nu \leq r)$ de sorte que $x_{m-\nu}=-x_{m+1+\nu}$ $(0 \leq \nu \leq m)$, avec la convention $x_{0}=1, x_{r+1}=-1$. Cette symétrie des zéros de $T_{r}$ nous sera utile plus loin et c'est pour l'imposer que nous avons choisi $r$ pair. La relation $T_{r+1}(x)+T_{r-1}(x)=2 x T_{r}(x)$ permet de montrer facilement par récurrence sur $r$ que

$$
l\left(T_{r}\right) \ll(1+\sqrt{2})^{r} .
$$

Nous définissons les polynômes $R$ et $S$ comme les uniques polynômes de degré $n$ satisfaisant à

$$
\begin{aligned}
& R\left(x_{\nu}\right)=\left\{\begin{array}{ll}
1 & (1 \leq \nu \leq m-1), \\
0 & (m \leq \nu \leq r),
\end{array} \quad R^{\prime}\left(x_{\nu}\right)=0 \quad(\nu \neq m),\right. \\
& S\left(x_{\nu}\right)=\left\{\begin{array}{ll}
1 & (1 \leq \nu \leq m+1), \\
0 & (m+2 \leq \nu \leq r),
\end{array} \quad S^{\prime}\left(x_{\nu}\right)=0 \quad(\nu \neq m+1) .\right.
\end{aligned}
$$

Les deux premières propriétés de (4.2) sont alors vérifiées et on a en fait

$$
R(z) \leq Y(z) \leq S(z) \quad(z \in \mathbb{R}) .
$$


(Voir par exemple le lemme 2.1 de [14] ou la démonstration du théorème II.7.10 de [21].)

Pour établir la troisième propriété, nous remarquons, grâce à la symétrie des zéros de $T_{r}(x)$ observée plus haut, que l'on a identiquement

$$
R(x)=1-S(-x)
$$

et donc que $V(x):=S(x)-R(x)=S(x)+S(-x)-1$ est pair, de degré $n$, et satisfait à

$$
V\left(x_{\nu}\right)=V^{\prime}\left(x_{\nu}\right)=0 \quad(\nu \neq m, m+1), \quad V\left(x_{m}\right)=V\left(x_{m+1}\right)=1 .
$$

On en déduit l'existence de nombres réels $\alpha_{n}$ et $\beta_{n}$ tels que

$$
V(x)=\left\{\alpha_{n}\left(x^{2}-x_{m}^{2}\right)+\beta_{n}\right\} \prod_{\nu=1}^{m-1}\left(x^{2}-x_{\nu}^{2}\right)^{2} .
$$

La relation $V\left(x_{m}\right)=1$ implique $\beta_{n}>0$, alors que la positivité du coefficient dominant de $S(x)$ fournit $\alpha_{n}>0$. Comme $x_{m} \sim \pi / 2 r$ lorsque $n \rightarrow \infty$, on a certainement

$$
\beta_{n}=\frac{4^{r} x_{m}^{2}}{T_{r}^{\prime}\left(x_{m}\right)^{2}} \sim \frac{4^{r-1} \pi^{2}}{r^{4}} \quad(n \rightarrow \infty),
$$

où nous avons utilisé la relation

$$
T^{\prime}\left(x_{m}\right)=\frac{r \sin r u_{m}}{\sin u_{m}}= \pm \frac{r}{\sin u_{m}} \sim \pm r \quad(r \rightarrow \infty)
$$

avec $u_{m}=\pi\left(m-\frac{1}{2}\right) / r$. De plus, la minoration $V(0) \geq Y(0)-Y(0-)=1$ fournit $\beta_{n}-\alpha_{n} x_{m}^{2}>0$, d'où

$$
\alpha_{n} \ll 4^{r} / r^{2} .
$$

Les estimations (4.3) et (4.4) nous permettent de donner une majoration de $|V(x)| \operatorname{sur}[-1,1]$. On a d'abord

$$
V(x) \ll \frac{4^{r-1}}{r^{4}} \prod_{\nu=1}^{m-1} x_{\nu}^{4}=\frac{T_{r}(0)^{2}}{r^{4} x_{m}^{4}} \ll 1 \quad\left(|x|<\left|x_{m-1}\right|\right) .
$$

Ensuite, en écrivant

$$
V(x)=T_{r}^{2}(x) \frac{\alpha_{n}\left(x^{2}-x_{m}^{2}\right)+\beta_{n}}{4^{r-1}\left(x^{2}-x_{m}^{2}\right)^{2}} .
$$

et en remarquant que

$$
x^{2}-x_{m}^{2} \asymp(m-\nu)^{2} / r^{2} \quad\left(1 \leq \nu \leq m-1, x_{\nu} \leq x \leq x_{\nu-1}\right),
$$

on obtient

$$
V(x) \ll 1 /(m-\nu)^{2} \quad\left(1 \leq \nu \leq m-1, x_{\nu} \leq|x| \leq x_{\nu-1}\right) .
$$


On peut maintenant établir la troisième propriété de (4.2). On a

$$
\begin{aligned}
\int_{-1}^{1} V(z) \mathrm{d} F(z) & =\sum_{\nu=1}^{r+1} \int_{x_{\nu-1}}^{x_{\nu}} V(z) \mathrm{d} F(z) \\
& \leq \sum_{\nu=1}^{r+1} \sup _{t \in\left[x_{\nu-1}, x_{\nu}\right]}\{V(t)\} \int_{x_{\nu-1}}^{x_{\nu}} \mathrm{d} F(z) \\
& \ll Q_{F}\left(x_{m-1}-x_{m+2}\right)+\sum_{1 \leq \nu \leq m-1} \frac{Q_{F}\left(x_{\nu-1}-x_{\nu}\right)}{(m-\nu)^{2}}
\end{aligned}
$$

d'après (4.5), (4.6) et la parité de $V$. On remarque que $x_{\nu-1}-x_{\nu} \ll 1 / n$, et donc, d'après (4.1),

$$
Q_{F}\left(x_{\nu-1}-x_{\nu}\right) \ll Q_{F}(1 / n) \quad(1 \leq \nu \leq r+1) .
$$

Cela termine la démonstration.

LEMME 2. Il existe une constante $A>0$ telle que, pour tout entier $n$ et tout nombre réel $T$ satisfaisant à $0<T \leq \frac{1}{3} n$, il existe des polynômes $P, Q$, de degrés $\leq n$, satisfaisant à

$$
\left\{\begin{array}{l}
P\left(\mathrm{e}^{-z / n}\right) \leq Y(z) \leq Q\left(\mathrm{e}^{-z / n}\right) \quad(z \in \mathbb{R}), \\
\int_{-T}^{T}\left\{Q\left(\mathrm{e}^{-z / n}\right)-P\left(\mathrm{e}^{-z / n}\right)\right\} \mathrm{d} G(z) \ll Q_{G}\left(\frac{T}{n}\right), \\
l(P)+l(Q) \leq(A n / T)^{n},
\end{array}\right.
$$

pour toute fonction croissante $G$ définie sur $\mathbb{R}$.

DÉmonstration. Soient $R, S$ les polynômes construits au Lemme 1 avec $f=Y$. On pose $P(z):=R(n(1-z) / 2 T)$ et $Q(z):=S(n(1-z) / 2 T)$. Alors

$$
l(P)+l(Q) \leq(A n / T)^{n} .
$$

Il résulte de plus de la première relation de (4.2) que

$$
P\left(\mathrm{e}^{-z / n}\right) \leq Y(z) \leq Q\left(\mathrm{e}^{-z / n}\right) \quad(z \in \mathbb{R}) .
$$

Posons alors

$$
F(z):=G\left(n \log \left(\frac{1}{1-2 T z / n}\right)\right) \quad(-1 \leq z \leq 1) .
$$


On a

$$
\begin{aligned}
\int_{-T}^{T}\{ & \left.Q\left(\mathrm{e}^{-z / n}\right)-P\left(\mathrm{e}^{-z / n}\right)\right\} \mathrm{d} G(z) \\
& =\int_{-T}^{T}\left\{S\left(\frac{n\left(1-\mathrm{e}^{-z / n}\right)}{2 T}\right)-R\left(\frac{n\left(1-\mathrm{e}^{-z / n}\right)}{2 T}\right)\right\} \mathrm{d} G(z) \\
& \leq \int_{-1}^{1}\{S(w)-R(w)\} \mathrm{d} F(w) \\
& \ll Q_{F}\left(\frac{1}{n}\right) \ll Q_{G}\left(\frac{T}{n}\right)
\end{aligned}
$$

grâce au Lemme 1. Cela fournit l'estimation centrale de (4.7) et achève la démonstration.

Lemme 3. Soit $G$ une fonction de répartition telle que

$$
h_{G}(u):=\widehat{G}(u)+\widehat{G}(-u)<\infty \quad(|u| \leq L) .
$$

Alors on a

$$
Q_{G}(1 / u) \sqrt{h_{G}(u)} \geq \frac{4}{15 \sqrt{3}} \quad(0<u \leq L)
$$

DÉMONSTRATION. On a

$$
\int_{\mathbb{R}} z^{2} \mathrm{~d} G(z) \leq \frac{1}{u^{2}} \int_{\mathbb{R}}\left\{\mathrm{e}^{z u}+\mathrm{e}^{-z u}\right\} \mathrm{d} G(z)=\frac{h_{G}(u)}{u^{2}} .
$$

On en déduit que, pour $T:=\sqrt{3 h_{G}(u)} / u$,

$$
\int_{|z|>T} \mathrm{~d} G(z) \leq \int_{\mathbb{R}} \frac{z^{2}}{T^{2}} \mathrm{~d} G(z) \leq \frac{h_{G}(u)}{T^{2} u^{2}}=\frac{1}{3} .
$$

Ainsi, $G(T)-G(-T) \geq \frac{2}{3}$. Comme l'intervalle $[-T, T]$ peut être recouvert par au plus $\lceil 2 T u\rceil \leq \frac{5}{2} \sqrt{3 h_{G}(u)}$ intervalles de longueur $1 / u$, on obtient bien (4.8).

5. Démonstration du théorème. La méthode employée par Alladi repose sur l'inégalité de Berry-Esseen: il déduit de l'hypothèse (i) une estimation de la différence des moments, qu'il utilise ensuite pour majorer la différence des transformées de Fourier. Nous procédons directement en adaptant à la situation présente la technique imaginée par Karamata [13] et rendue effective par Freud [11] pour établir le théorème taubérien de Hardy-Littlewood.

On observe d'abord que, pour $-L \leq u \leq L, T>0$,

$$
\int_{|z|>T / 2} \mathrm{~d} F(z) \leq \int_{\mathbb{R}} \mathrm{e}^{|u z|-u T / 2} \mathrm{~d} F(z) \ll \mathrm{e}^{-u T / 2} h(u) .
$$


Cette majoration est également valable pour $G$. On peut donc écrire

$$
\|F-G\|_{\infty} \ll \sup _{|w| \leq T / 2}|F(w)-G(w)|+\mathrm{e}^{-u T / 2} h(u) \quad(|u| \leq L, T>0) .
$$

Soient $w \in\left[-\frac{1}{2} T, \frac{1}{2} T\right]$ et $n \geq \frac{3}{2} \kappa T$. En appliquant le Lemme 2 avec $\frac{1}{2} \kappa T$ à la place de $T$ et $z-w$ à la place de $z$, on obtient, grâce à (4.1), l'existence d'une constante $b=b(\kappa)>0$ et de polynômes $P$ et $Q$, de degrés n'excédant pas $n$, tels que

$$
\left\{\begin{array}{l}
P\left(\mathrm{e}^{-\kappa(z-w) / 2 n}\right) \leq Y(z-w) \leq Q\left(\mathrm{e}^{-\kappa(z-w) / 2 n}\right) \quad(|z-w| \leq T), \\
\int_{w-T}^{w+T}\left\{Q\left(\mathrm{e}^{-\kappa(z-w) / 2 n}\right)-P\left(\mathrm{e}^{-\kappa(z-w) / 2 n}\right)\right\} \mathrm{d} G(z) \ll_{\kappa} Q_{G}\left(\frac{T}{n}\right), \\
l(P)+l(Q) \ll\left(\frac{b n}{T}\right)^{n}
\end{array}\right.
$$

On peut maintenant écrire

$$
\begin{aligned}
F(w+T)-F(w) & =\int_{w-T}^{w+T} Y(z-w) \mathrm{d} F(z) \\
& \leq \int_{w-T}^{w+T} Q\left(\mathrm{e}^{-\kappa(z-w) / 2 n}\right) \mathrm{d} F(z) \\
& \leq \int_{\mathbb{R}} Q\left(\mathrm{e}^{-\kappa(z-w) / 2 n}\right) \mathrm{d} F(z)+Z_{n}(w, T)
\end{aligned}
$$

avec

$$
Z_{n}(w, T):=\int_{|z-w|>T}\left|Q\left(\mathrm{e}^{-\kappa(z-w) / 2 n}\right)\right| \mathrm{d} F(z) .
$$

L'hypothèse (i) nous permet d'estimer le premier terme du membre de droite de (5.4): on a

$$
\int_{\mathbb{R}} Q\left(\mathrm{e}^{-\kappa(z-w) / 2 n}\right) \mathrm{d} F(z)=\int_{\mathbb{R}} Q\left(\mathrm{e}^{-\kappa(z-w) / 2 n}\right) \mathrm{d} G(z)+O\left(l(Q) \varepsilon \mathrm{e}^{\kappa T / 2}\right) .
$$

Nous majorons $Z_{n}(w, T)$ en faisant appel à (ii). Introduisons un paramètre $v$, $\frac{1}{2} \kappa \leq v \leq L$, et observons que les conditions $|z-w|>T$ et $|w| \leq \frac{1}{2} T$ impliquent

$$
\frac{1}{2} \kappa|z-w| \leq \frac{1}{2} \kappa|z|+\frac{1}{4} \kappa T+\left(v-\frac{1}{2} \kappa\right)\left(|z|-\frac{1}{2} T\right)=v|z|-\frac{1}{2} v T+\frac{1}{2} \kappa T .
$$

Il suit

$$
\begin{aligned}
Z_{n}(w, T) & \leq l(Q) \mathrm{e}^{\frac{1}{2} \kappa T-\frac{1}{2} v T} \int_{\mathbb{R}} \mathrm{e}^{v|z|} \mathrm{d} F(z) \\
& \ll l(Q) \mathrm{e}^{\frac{1}{2} \kappa T-\frac{1}{2} v T} h(v) .
\end{aligned}
$$

En reportant dans (5.4), en appliquant (5.1) avec $u=v$ pour estimer $1-F(w+T)$ et en tenant compte de (5.5), nous obtenons, pour une constante 
absolue convenable $K$,

$$
\begin{aligned}
1-F(w) \leq & \int_{\mathbb{R}} Q\left(\mathrm{e}^{-\kappa(z-w) / 2 n}\right) \mathrm{d} G(z) \\
& +K \mathrm{e}^{\kappa T / 2}\{l(Q)+1\}\left\{\varepsilon+\mathrm{e}^{-v T / 2} h(v)\right\} .
\end{aligned}
$$

Un calcul symétrique fournit la minoration

$$
\begin{aligned}
1-F(w) \geq & \int_{\mathbb{R}} P\left(\mathrm{e}^{-\kappa(z-w) / 2 n}\right) \mathrm{d} G(z) \\
& -K \mathrm{e}^{\kappa T / 2}\{l(P)+1\}\left\{\varepsilon+\mathrm{e}^{-v T / 2} h(v)\right\},
\end{aligned}
$$

et les inégalités (5.7) et (5.8) sont également valables pour $G(w)$. En reportant dans (5.2) avec $u=v$, nous pouvons donc écrire

$$
\|F-G\|_{\infty} \ll \sup _{|w| \leq T / 2} I_{n}(w, T)+\left\{\varepsilon+\mathrm{e}^{-v T / 2} h(v)\right\} \mathrm{e}^{\kappa T / 2}\left(\frac{b n}{T}\right)^{n},
$$

avec

$$
\begin{aligned}
I_{n}(w, T) & :=\int_{\mathbb{R}}(Q-P)\left(\mathrm{e}^{-\kappa(z-w) / 2 n}\right) \mathrm{d} G(z) \\
& \ll Q_{G}(T / n)+\int_{|z-w|>T}(Q-P)\left(\mathrm{e}^{-\kappa(z-w) / 2 n}\right) \mathrm{d} G(z) .
\end{aligned}
$$

Un raisonnement identique à celui qui fournit (5.6) nous permet de montrer que la dernière intégrale est

$$
\ll\{l(P)+l(Q)\} \mathrm{e}^{\frac{1}{2} \kappa T-\frac{1}{2} v T} h(v) .
$$

En reportant dans (5.9), il s'ensuit que

$$
\|F-G\|_{\infty} \ll Q_{G}\left(\frac{T}{n}\right)+\left\{\varepsilon+\mathrm{e}^{-v T / 2} h(v)\right\} \mathrm{e}^{\kappa T / 2}\left(\frac{b n}{T}\right)^{n} .
$$

Nous sommes maintenant en mesure d'établir la majoration (1.4). Nous pouvons pleinement supposer $\varepsilon$ assez petit et $L$ assez grand, en fonction de $\kappa$ et des constantes implicites mentionnées dans l'énoncé. Cela implique que $v$ peut être choisi arbitrairement grand, disons $v \geq v_{0}$. Choisissons alors $v:=\min (L, W)$ où $W:=h^{-1}(1 / \varepsilon)$, ainsi qu'il est précisé dans l'énoncé, et posons

$$
T:=4 \frac{\log h(v)}{v}, \quad n:=\left[\frac{\log h(v)}{3 \log v}\right] .
$$

L'hypothèse (1.3) implique $n \geq 1$ et $n \geq \frac{3}{2} \kappa T$ pour $v_{0}$ assez grand. De plus, on peut écrire, grâce à (4.1),

$$
Q_{G}\left(\frac{T}{n}\right) \ll Q_{G}\left(\frac{\log v}{v}\right) \ll Q_{G}\left(\frac{\log L}{L}+\frac{\log W}{W}\right) .
$$


Il reste à montrer que le second terme du membre de droite de (5.10) peut être englobé par cette dernière majoration. On a

$$
\mathrm{e}^{-(v-\kappa) T / 2} h(v)=h(v)^{-1+2 \kappa / v}, \quad \varepsilon \mathrm{e}^{\kappa T / 2}=\varepsilon h(v)^{2 \kappa / v} \leq h(v)^{-1+2 \kappa / v},
$$

et

$$
\left(\frac{b n}{T}\right)^{n} \leq h(v)^{\frac{1}{3} \log (b v / \log v) / \log v} \ll h(v)^{\frac{1}{3}}
$$

Le terme complémentaire de la majoration (5.10) est donc $\ll h(v)^{-1 / 2}$ dès que $v$ est assez grand. Le Lemme 3 permet de conclure.

\section{BIBLIOGRAPHIE}

[1] AlLADI, K. (1985). A study of the moments of additive functions using Laplace transform and sieve methods. Proceedings of the Fourth Conference on Number Theory. Lecture Notes in Math. 1122 1-37. Springer, Berlin.

[2] Alladi, K. (1987). An Erdős-Kac theorem for integers free of large prime factors. Acta Arithmetica 49 81-105.

[3] BERRY, A. C. (1941). The accuracy of the Gaussian approximation to the sum of independent variates. Trans. Amer. Math. Soc. 49 122-136.

[4] Delange, H. (1959). Sur des formules dues à Atle Selberg. Bull. Sci. Math. (2) 83 101-111.

[5] Elliott, P.D.T.A. (1980). Probabilistic Number Theory I. Springer, New York.

[6] ERDős, P. (1946). On the distribution function of additive functions. Ann. Math. 47 1-20.

[7] ERDős, P. and KAC, M. (1939). On the Gaussian law of errors in the theory of additive functions. Proc. Nat. Acad. Sci. U.S.A. 25 206-207.

[8] ERDős, P. and KAC, M. (1940). The Gaussian law of errors in the theory of additive number theoretic functions. Amer. J. Math. 62 738-742.

[9] Esseen, C. G. (1945). Fourier analysis of distribution functions. A mathematical study of the Laplace-Gaussian law. Acta Math. 77 1-125.

[10] Feller, W. (1971). An Introduction to Probability Theory and Its Applications 2, 2nd ed. Wiley, New York.

[11] Freud, G. (1952). Restglied eines Tauberschen Sätzes I. Acta Math. Acad. Sci. Hungar. 2 299-308.

[12] Hall, R. R. and Tenenbaum, G. (1988). Divisors. Cambridge Univ. Press.

[13] KARAmata, J. (1931). Neuer Beweis und Verallgemeinerung der Tauberschen Sätze, welche die Laplacesche und Stieltjesche Transformation betreffen. J. Reine Angew. Math. 164 $27-39$.

[14] KorevaAR, J. (1954). A very general form of Littlewood's theorem. Indag. Math. 16 36-45.

[15] LeVeque, W. J. (1949). On the size of certain number-theoretic functions. Trans. Amer. Math. Soc. 66 440-463.

[16] Maier, H. and Tenenbaum, G. (1984). On the set of divisors of an integer. Invent. Math. 76 121-128.

[17] Petrov, V. V. (1975). Sums of Independent Random Variables. Springer, Berlin.

[18] RÉNYI, A. and TuRÁN, P. (1958). On a theorem of Erdős-Kac. Acta Arith. 4 71-84.

[19] STEF, A. (1992). L'ensemble exceptionnel dans la conjecture d'Erdős concernant la proximité des diviseurs. Doctorat d'université, Département de mathématiques de l'Université Henri-Poincaré Nancy 1.

[20] Stef, A. and Tenenbaum, G. (1998). Entiers lexicographiques. Ramanujan J. 2 167-184. 
[21] Tenenbaum, G. (1995). Introduction à la théorie analytique et probabiliste des nombres, Cours Spécialisés, no 1, Société Mathématique de France, Paris. [Traduction en anglais (1995). Introduction to Analytic and Probabilistic Number Theory. Cambridge Univ. Press.]

[22] TuRán, P. (1934). Az egész számok primosztóinak számáról. Mat. és. Fiz. Lapok 41 103-130.

INSTITUT ÉLIE CARTAN

UNIVERSITÉ HeNRI POINCARÉ-NANCY 1

BP 239

54506 VANDGEURE CEDEX

FRANCE

E-MAIL: stef@iecn.u-nancy.fr tenenb@ciril.fr 